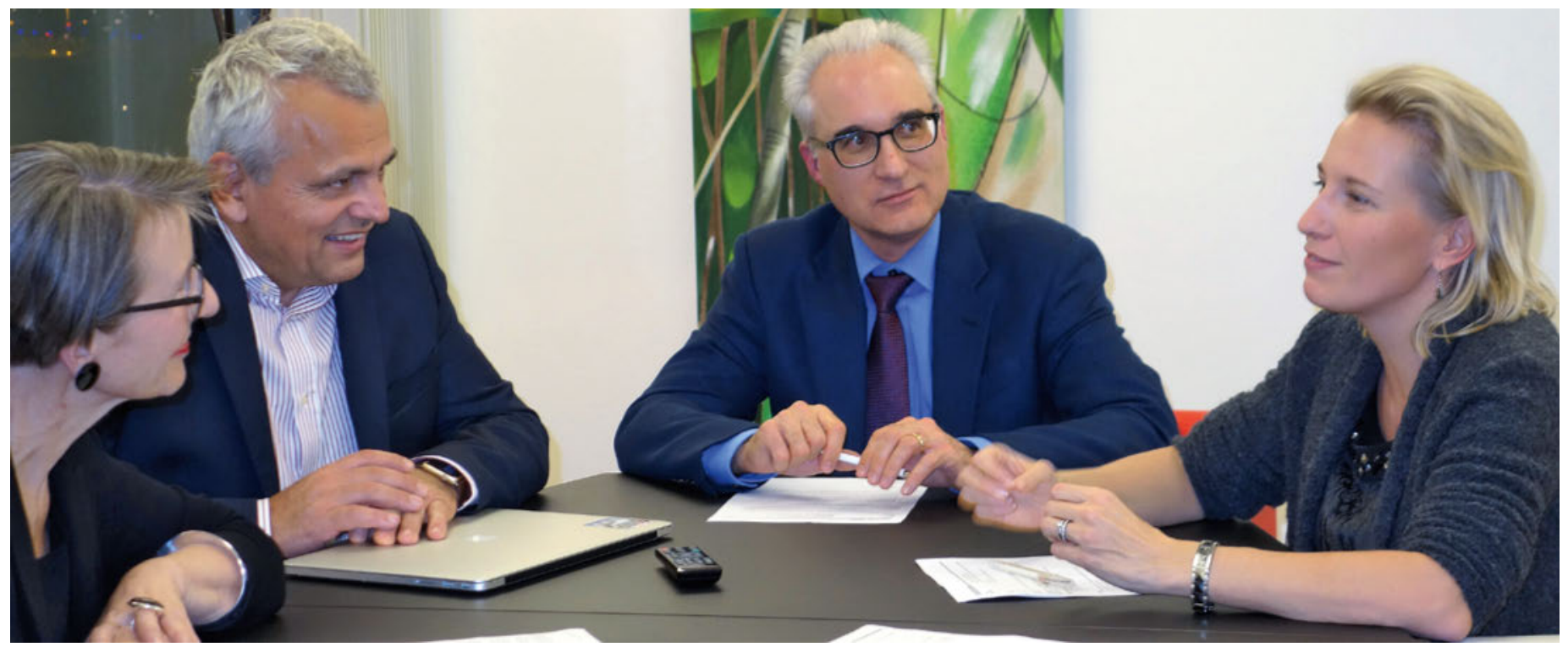

Was ist "Lean»? Rebecca Spirig, Daniel Walker, Carsten Viehl und Katharina Rüther-Wolf (von links) diskutierten gemeinsam mit unserer Autorin Anna Sax über ein Management-Tool, das ursprünglich von Toyota zur Prozessoptimierung eingeführt wurde und mittlerweile auch in Spitälern zur Anwendung kommt.

\title{
"Wo die Standardisierung aufhört, fängt das Denken an»
}

\section{Anna Sax}

Lic. oec. publ., MHA, Mitglied der Redaktion

Ein Management-System aus der Industrie gibt in den Spitälern zurzeit viel zu reden. Unter dem Titel «Lean Hospital» werden Prozesse optimiert, standardisiert und rund um die Patientinnen und Patienten herum neu organisiert. Die Schweizerische Ärztezeitung wollte im Round-Table-Gespräch mit vier Expertinnen und Experten genauer erfahren, worum es bei «Lean» geht.

Was ist «Lean»? Ist es eine Philosophie, eine Kultur, oder einfach nur eine Management-Methode? Was meinen Sie als Expertinnen und Experten, wenn Sie von «Lean Hospital» sprechen?

Daniel Walker: «Lean» ist ein Management-System, das wirksame Instrumente zusammenfasst. Es ist faszinierend, weil es funktioniert. Alle kommen damit ziemlich sicher ins Ziel. Es funktioniert in verschiedener Hinsicht: um etwas zum Guten zu verändern für die Patientinnen und Patienten oder um Führung wirksamer zu machen. Mit «Lean Hospital» ist es möglich, ein Spital als Ganzes weiterzuentwickeln.

Rebecca Spirig: Wenn ich höre, dass «Lean» eine Philosophie sei, dann muss ich schmunzeln. Ich bin gerne damit einverstanden, dass "Lean» eine Methodik ist, vielleicht auch ein System, aber bestimmt keine Philosophie. «Lean» stellt ein ganzes Set von Methoden zur

\section{Die Gesprächsteilnehmer}

Katharina Rüther-Wolf, Dr. med. MBA, Medical-Process Managerin, Universitätsspital Basel

Rebecca Spirig, Prof. Dr., Direktorin Pflege und MTTB, UniversitätsSpital Zürich

Carsten T. Viehl, Prof. Dr. med., ärztlicher Leiter Departement Chirurgie, Spitalzentrum Biel

Daniel Walker, lic. phil, EMBA HSG, Managing Partner

walkerprojekt ag 
Verfügung, und wir haben Beispiele, die ihre Wirksamkeit zeigen.

Katharina Rüther-Wolf: Für mich ist «Lean» mehr als eine Methode. Es ist die Summe von Methode, Kultur- und Führungsveränderung. Meine Beschreibung würde so lauten: «Lean» ist die bewusste Investition von Zeit und Brainpower aller Mitarbeitenden mit dem Ziel, die Unternehmensleistung für die Patienten und ihre Angehörigen zu verbessern.

\section{«Für mich ist 'Lean' mehr als eine Methode.»}

Carsten Viehl: Für mich ist «Lean» in erster Linie ein Mindset: Zuerst einigen wir uns darauf, in welche Richtung wir denken, und das setzen wir dann mit einer bestimmten Methode um.

\section{Bessere und effizientere Spitäler, Patientinnen und Patienten im Zentrum, das sind keine neuen Postu- late. Was ist neu an "Lean Hospital»?}

Viehl: Neu ist, dass man es endlich einmal umsetzt. Man muss Mauern herunterreissen, sich gemeinsam hinsetzen und zum Beispiel eine Teamsprechstunde einführen.

\section{Was ist das, eine Teamsprechstunde?}

Viehl: Typischerweise geht ein Patient zuerst zum Hausarzt, dann vielleicht zur Spezialistin, dann ins Spital und dort wieder zu verschiedenen Stationen. Wir versuchen, wenigstens den Teil, der sich im Spital abspielt, neu zu gestalten und alle Beteiligten an einen Tisch zu bringen. Der Patient erzählt der Chirurgin einmal etwas, was die anderen Beteiligten direkt mitbekommen. Es ist erstaunlich, was sich damit alles verändert. Manchmal findet man so ohne grosse Worte eine gemeinsame Ebene. Das ist hocheffizient im Sinne des Patienten.

\section{Warum ist nicht schon früher jemand auf diese Idee gekommen?}

Spirig: Vieles von dem, was jetzt unter dem Titel «Lean" geschieht, hat schon länger existiert. Wir haben immer wieder Prozesse neu aufgegleist und verbessert. Jetzt werden die verschiedenen Methoden aber neu gebündelt, das Wort «Mindset» trifft es gut. Wir haben «Lean»-Methoden in verschiedenen Projekten angewendet und gesehen, dass vieles am Schluss ganz klar den Patientinnen und Patienten zugutekommt.

\section{Was haben Sie konkret gemacht?}

Spirig: Wir haben für unsere Pflegeteams, und ich spreche von 75 Teams, den Skill-Grade-Mix auf die Pa- tientengruppen abgestimmt, für die sie Verantwortung tragen. Diese Anpassungen kombinierten wir mit dem Thema Verschwendung, schauten also genau hin, wie die Leute miteinander arbeiten. Wir führten «Gembas» durch, das heisst, wir folgten den Leuten, beobachteten sie und stellten ihnen dann ein Feedback zur Verfügung. Die Teams erarbeiteten selbst Lösungen. Wir sehen nun bei etwa der Hälfte der Teams, dass sie mit neuen Methoden arbeiten. Die andere Hälfte macht es nicht oder nicht mehr. «Lean» muss also regelmässig aufgefrischt werden, das ist nicht anders als bei anderen Prozessverbesserungen.

\section{Frau Rüther-Wolf, Sie sind verantwortlich für die Umsetzung von "Lean" im Universitätsspital Basel. Welche Ziele verfolgen Sie?}

Rüther-Wolf: Wir haben von der Spitalleitung den Auftrag, die drei Felder Qualität, Produktivität und Mitarbeitende parallel anzugehen. Hier liegt der Unterschied zu früheren Projekten: Da führten wir Projekte zur Prozessentwicklung durch mit dem Ziel, die Produktivität zu verbessern. Dann gab es Qualitätsprojekte, die Qualitätsmessungen nach sich zogen. Das führte teilweise zu Widersprüchen, man kam sich gegenseitig in die Quere. Mit «Lean» möchten wir die Produktivität und Qualität gleichzeitig verbessern. Das ist kein Widerspruch.

\section{Können Sie uns ein Beispiel geben für etwas, das sich} durch "Lean» in Ihrem Spital verändert hat?

Rüther-Wolf: Ein gutes Beispiel ist die präoperative Sprechstunde in der Augenklinik. Wir sahen, dass Pa-

«Die Schilder waren so beschriftet, dass man sie mit einer Sehbehinderung nicht lesen konnte.»

tientinnen und Mitarbeitende unzufrieden sind, und richteten unseren Fokus auf diese Sprechstunde. Drei Stunden verbrachten die Patienten durchschnittlich in der Klinik. Sie mussten 600 Meter weit laufen, teils über Treppen. Die Schilder waren so beschriftet, dass man sie mit einer Sehbehinderung nicht lesen konnte. Wir gingen das durch, nahmen Messungen vor zur benötigten Zeit und zur Zufriedenheit von Patienten und Personal. Aufgrund der Ergebnisse nahm das interprofessionelle Team der Mitarbeitenden mit unserer Unterstützung Veränderungen und Anpassungen vor. Heute geht es schneller, was zum einen die Patienten erfreut und was auch weniger Überstunden für die Mitarbeiter bedeutet. Alle Beteiligten sind deutlich zufriedener. 
Stehen nun die Patienten über allem? Erhalten sie individuellen Service, wird jeder Wunsch sofort erfüllt? Spielt es noch eine Rolle, wie es dem Personal geht? Walker: Im Grundsatz ist es einfach. «Lean» meint: Gib dem Patienten das, was er jetzt braucht. Wenn die Patientinnen und Patienten warten müssen und nicht die Medizin erhalten, die sie brauchen, weil das Personal gestresst ist, gehen am Schluss alle unzufrieden nach Hause. Wer im Spital arbeitet, tut das nicht wegen des Geldes, sondern aus einer inneren Überzeugung heraus. Wenn wir nun eine Situation schaffen, wo die Leute ruhiger arbeiten und dem Patienten das geben können, was er braucht, macht sie das glücklich. Das ist ihre Bestimmung, dafür haben sie langjährige Ausbildungen gemacht. Wenn sie auch noch ihre Qualität verbessern können, dann sind sie begeistert.

Sie sagen, die Leute in den Spitälern seien intrinsisch motiviert. Beim herrschenden Personalmangel können sie aber jederzeit den Job wechseln, wenn sie nicht zufrieden sind.

Walker: Das ist ja genau die Stärke von «Lean». Auf der "Lean»-Bettenstation in Liestal zum Beispiel hat die Pflegedienstleiterin eine Liste von Leuten, die bei ihr arbeiten möchten, sobald eine Stelle frei wird.

\section{Herr Viehl, können Sie uns weitere Beispiele aus Ihrem Wirkungsbereich geben?}

Viehl: Was wir im klinischen Alltag häufig erleben, sind sinnlose Schlaufen, klassischerweise, weil Prozesse nicht aufeinander abgestimmt sind. Oder sie sind aufeinander abgestimmt, aber zeitlich verschoben, oder die Informationsflüsse sind unvollständig oder nicht standardisiert. Genau diese Schlaufen führen zu Frustration: Wenn ich dem hohen Ethos entsprechen

"Was wir im klinischen Alltag häufig erleben, sind sinnlose Schlaufen, klassischerweise, weil Prozesse nicht aufeinander abgestimmt sind.»

und dem Patienten jetzt geben möchte, was er braucht, muss ich mich massiv einsetzen. Ich muss diverse Telefonate führen und Abgleichungen zwischen Leuten persönlich vornehmen. Die Abnützung, die sich mit der Zeit einstellt, führt zu Resignation, so dass ich es irgendwann nicht mehr mache. Wenn Sie nach einem Beispiel fragen: Ein grosser Fortschritt ist das Patientenboard auf den verschiedenen Stationen. Das ist eigentlich etwas völlig Simples, doch wir vermeiden damit Informationsschlaufen und stellen Verbindlichkeit und Übersicht her. Patientinnen und Patienten sind begeistert. Sie und ihre Angehörigen können selber nachschauen, was die nächsten Schritte und die Vereinbarungen sind.

Wie begeistert die Patientinnen sind und wie zufrieden das Personal ist, lässt sich mit Befragungen ermitteln. Aber das ist noch keine umfassende Evaluation. Kann man zum Beispiel auch etwas über den Patientennutzen und die Qualitätsentwicklung sagen?

Spirig: Das kann man. Wir haben bei unserem SkillGrade-Mix-Projekt auf verschiedenen Ebenen gemessen

"Die Visite ist in den meisten Häusern, auch bei uns, ein schlecht abgestimmter interprofessioneller Prozess.»

und auch qualitative Interviews durchgeführt. Etwas, woran wir intensiv gearbeitet haben, ist der Visitenprozess. Die Visite ist in den meisten Häusern, auch bei uns, ein schlecht abgestimmter interprofessioneller Prozess, bei dem viel Zeit und Energie für Nicht-Zusammenarbeit verlorengeht. Das konnten wir nun in verschiedenen Schlaufen und an verschiedenen Orten verbessern. Interprofessionelle Personal- und Patientenbefragungen haben überzeugende Resultate ergeben. Rüther-Wolf: Auf der «Lean»-Station leistet das Personal weniger Überzeit. Es wird auch weniger geklingelt, die Durchlauf- und Wartezeiten sind geringer, und deshalb sind die Patientinnen und Patienten besser gelaunt. Die Kennzahlen sprechen hier eine eindeutige Sprache. Das Einzige, was wir noch nicht geschafft haben, ist, die medizinische Qualität zu messen. Es gibt Hinweise in der Literatur aus den USA, dass sich auch die Qualität verbessert. Wir sind hier noch nicht weit genug.

Das Spitalmanagement hört gern, was Sie bisher festgestellt haben: Mit "Lean" wird das Spital produktiver, die Patientenzufriedenheit steigt und man findet leichter Personal. Was tun medizinische und pflegerische Kaderleute, um sich nicht unter dem Titel «Lean Hospital» für betriebswirtschaftliche Ziele instrumentalisieren zu lassen?

Viehl: Wir müssen klarstellen, dass «Lean» kein Mittel ist, um Personal abzubauen. Die Kräfte, die durch Effizienzgewinne freigespielt werden, müssen wir darauf verwenden, den Verbesserungsprozess weiter voranzutreiben. Wenn man dieses Commitment am Anfang nicht abgibt, hat man Mühe, die Mitarbeitenden zu überzeugen, weil sie dann eben Angst haben, ihre eigenen Arbeitsplätze abzuschaffen.

Spirig: Es hat sehr viel mit Kultur zu tun. Wir arbeiten im USZ kennzahlenorientiert, sowohl Top-down wie 
auch Bottom-up. Wir nennen es nicht «Lean», denn unsere Kaderleute haben mit "Lean" in ihrer Wahrnehmung schwierige Erfahrungen gemacht. Hingegen können sie mit Feedback und Kennzahlen hervorragend umgehen, das ist ihre Welt.

\section{Wir haben jetzt viel von Prozessen und Standardisie- rungen gesprochen. Wo braucht es das und wo gibt es Grenzen?}

Spirig: Wir sind dabei, unsere Standardprozesse zu verbessern. Ich habe vorhin die Visite erwähnt. Dort braucht es Prozessmanagement-Strategien. Im ganz direkten medizinisch- oder pflegerisch-diagnostischen Prozess mit den Patientinnen und Patienten hingegen ist «Lean» ein Instrument, das ich nicht unterstütze.

Walker: Das finde ich spannend. Ich wüsste gern von dir, Carsten, welches Potential es in der Medizin gibt.

\section{«Man soll dort standardisieren, wo etwas standardisierbar ist.»}

Viehl: Was Rebecca gesagt hat, scheint mir interessant zu sein. Daniel und ich haben lange darüber diskutiert. Du bist ja der Meinung, Daniel, man könne alles standardisieren, und teilweise hast du mich überzeugt. Andererseits wird ärztliches und pflegerisches Handeln auch als Kunst bezeichnet, und das kann nicht immer nach dem Büchlein laufen. Aber das ist nicht zwingend ein Widerspruch. Man soll dort standardisieren, wo etwas standardisierbar ist, zum Beispiel an den Schnittstellen arbeiten wie dem ungeordneten Ablauf der Visiten. Dann brauchen wir aber auch Raum für das «Künstlerische», für das Unvorhergesehene. Oder einfach zum Denken.

Rüther-Wolf: Die sinnvolle Standardisierung mit «Lean» gibt den Freiraum, den wir brauchen für die komplexen und aussergewöhnlichen Fälle, die viel Flexibilität verlangen.

\section{Stichworte zu "Lean Hospital»}

Die Prinzipien von "Lean Management» stammen ursprünglich von Toyota. Seit einigen Jahren dienen die schlanken Produktionsprozesse auch in Schweizer Spitälern als Vorbild. Bei «Lean Hospital» geht es um Prozessoptimierungen, klarere Absprachen zwischen den beteiligten Fachbereichen und mehr Verbindlichkeit und Transparenz gegenüber den Patientinnen und Patienten. Letztere sollen schneller und in besserer Qualität die Leistungen erhalten, die sie brauchen. Elemente von "Lean" sind beispielsweise Patientenboards in allen Patientenzimmern, Kaizen-Tafeln, wo das Personal Verbesserungsvorschläge öffentlich anbringen kann, oder mobile Pflegewagen, damit die Dokumentation direkt am Patientenbett erfolgen kann.

Die Begrifflichkeiten aus der Industrie und der Fokus auf Produktivität und Effizienz stossen bei Ärzteschaft und Pflegenden teilweise auf Unverständnis. Die Auseinandersetzungen drehen sich darum, ob sich Gesundheitsversorgung an betriebswirtschaftlichen Methoden orientieren darf, wie weit die Abläufe in einem Spital standardisierbar sind, welcher Nutzen für die Patientinnen und Patienten wirklich herausschaut und wie es dem Gesundheitspersonal dabei geht.

Walker: Ich war kürzlich im Memorial Sloan Kettering Cancer Center, einem der weltweit führenden TumorZentren. Dort haben sie extrem viel standardisiert. Sie sagen: «Wir haben so viel Brain verschwendet, indem wir Dinge, die klar sind und wo wir uns absolut einig waren, jedes Mal wieder neu erfunden haben.» Heute geht alles viel schneller. Sie können dem Patienten innerhalb von 36 Stunden einen Behandlungsplan auf den Tisch legen, die Qualität ist besser als vorher. Das haben sie über Standardisierung hingekriegt. Diese gibt eine sichere Basis, und dann kommt der Punkt, wo der Patient individuell ist. Hier fängt das Denken an.

\section{Literatur}

Walker D, Herausgeber. Lean Hospital. Das Krankenhaus der Zukunft. Berlin: MWV Medizinisch Wissenschaftliche Verlagsgesellschaft; 2015.

\section{Bildnachweis}

Anna Sax 